\title{
SOME REMARKS ON MODULARITY OF THE CONGRUENCE LATTICE OF REGULAR $\omega$-SEMIGROUPS
}

\section{BONZINI and A. CHERUBINI}

(Received 14 April 1992; revised 10 August 1992)

\author{
Communicated by P. G. Trotter
}

\begin{abstract}
In this paper conditions of $M$-symmetry, strong, semimodularity and $\theta$-modularity for the congruence lattice $L(S)$ of a regular $\omega$-semigroup $S$ are studied. They are proved to be equivalent to modularity. Moreover it is proved that the kernel relation is a congruence on $L(S)$ if and only if $L(S)$ is modular, generalizing an analogous result stated by Petrich for bisimple $\omega$-semigroups.
\end{abstract}

1991 Mathematics subject classification (Amer. Math. Soc.): $20 \mathrm{M} 10$.

Keywords and phrases: $M$-symmetric lattice, strongly semimodular lattice, semimodular lattice, $\theta$ modular lattice, double covering property, kernel relation, regular $\omega$-semigroup.

\section{Introduction}

In [3] and [4] the authors stated conditions under which the lattice of congruences $L(S)$ of a regular $\omega$-semigroup $S$ is respectively modular or semimodular. Two other conditions, $M$-symmetry and strong semimodularity, are usually examined for the congruence lattice of semigroups. So it is natural to ask for conditions implying $M$-symmetry and strong semimodularity for the congruence lattice of regular $\omega$ semigroups.

We remark that we use terminology consistent with that used by Mitsch in [6]. Following other authors, semimodularity and strong semimodularity are called, respectively, double covering property and semimodularity. It is well known that in every lattice modularity implies $M$-symmetry, $M$-symmetry implies strong semimodularity, which in turn implies semimodularity. Thus we begin with conditions for strong semimodularity. Firstly, we give a characterization of regular $\omega$-semigroups

Work supported by M.U.R.S.T. and C.N.R.

(C) 1995 Australian Mathematical Society 0263-6115/95 $\$ A 2.00+0.00$ 
whose congruence lattice is strongly semimodular. Then we prove that this condition is equivalent to the modularity conditions given in [3]. Hence this paper becomes a revisit of regular $\omega$-semigroups with modular lattice of congruences.

Petrich introduced two relations on the congruence lattice of a regular semigroup: the trace relation and the kernel relation. In general the latter one is not a congruence on the congruence lattice, so he studied conditions in order that the kernel relation $\mathscr{K}$ is a congruence and in $[8,9]$ observed a strict connection between this fact and the modularity of the congruence lattice for classes of regular semigroups. We study the kernel relation for a regular $\omega$-semigroup and prove that $L(S)$ is modular if and only if $\mathscr{K}$ is a congruence on $L(S)$.

Finally we prove that the modularity of $L(S)$ is equivalent to $\theta$-modularity.

Notation and terminology will be as in [3] and [4], and a knowledge of these papers is useful in the following. As usual $\sigma$ denotes the least group congruence, $\mathscr{H}$ and $\mathscr{D}$ the Green's relations, $\iota$ the identity congruence on $S$, and $\mathbb{N}$ the set of non-negative integers.

\section{Section 1}

For sake of completeness, we begin by recalling the main results on the structure of regular $\omega$-semigroups and on the description of some their congruences.

DEFINITION 1.1 (see, for example, [10]). A regular $\omega$-semigroup $S$ is a regular semigroup whose set of idempotents $E(S)$, or shortly $E$, forms an $\omega$-chain

$$
e_{0}>e_{1}>\cdots>e_{n}>\cdots
$$

under the natural order defined on $E$ by the rule $e \geq f$ if and only if $e f=f=f e$.

For a regular $\omega$-semigroup, Munn [7] proved the following result:

THEOREM A. Let $S$ be a regular $\omega$-semigroup.

(i) If $S$ has no kernel, then it is the union of an $\omega$-chain of groups.

(ii) If the kernel of $S$ coincides with $S$, then $S$ is a simple regular $\omega$-semigroup.

(iii) If $S$ has a proper kernel, then $S$ is a (retract) ideal extension of a simple regular $\omega$-semigroup $K$ by a semigroup $H^{0}$, where $H$ is a finite chain of groups and $H^{0}$ is obtained from $H$ by adjoining a zero. Moreover this extension is determined by means of a homomorphism of $H$ into the group of units of $K$.

Thus, the following theorem completely determines the structure of regular $\omega$ semigroups: 
THEOREM B (Kocin [5] and Munn [7]). Let $d$ be a positive integer and let $\left\{G_{i} \mid\right.$ $i=0, \ldots, d-1\}$ be a set of $d$ pairwise disjoint groups. Let $\gamma_{d-1}$ be a homomorphism of $G_{d-1}$ into $G_{0}$ and, if $d>1$, let $\gamma_{i}$ be a homomorphism of $G_{i}$ into $G_{i+1}$ ( $i=$ $0, \ldots, d-2)$. For every $n \in \mathbb{N}$ let $\gamma_{n}=\gamma_{n(\bmod d)}$ where $n(\bmod d)$ denotes the integer equivalent to $n$ modulo $d$, belonging to $N$ and less than $d$. For $m, n \in \mathbb{N}$ and $m<n$ write

$$
\alpha_{m, n}=\gamma_{m} \gamma_{m+1} \ldots \gamma_{n-1}
$$

and for all $n \in N$ let $\alpha_{n, n}$ denote the identity automorphism of $G_{n(\bmod d)}$

Let $S$ be the set of ordered triples $\left(m, a_{i}, n\right)$, where $m, n \in \mathbb{N}, 0 \leq i \leq d-1$ and $a_{i} \in G_{i}$. Define a multiplication in $S$ by the rule

$$
\left(m, a_{i}, n\right)\left(p, b_{j}, q\right)=\left(m+p-r,\left(a_{i} \alpha_{u, w}\right)\left(b_{j} \alpha_{v, w}\right), n+q-r\right)
$$

where $r=\min \{n, p\}, u=n d+i, v=p d+j$ and $w=\max \{u, v\}$. Denote the so formed groupoid by $S\left(d, G_{i}, \gamma_{i}\right)$. Then $S\left(d, G_{i}, \gamma_{i}\right)$ is a simple regular $\omega$-semigroup with exactly $d \mathscr{D}$-classes and any regular $\omega$-semigroup is isomorphic to a semigroup $S\left(d, G_{i}, \gamma_{i}\right)$.

For $n \in \mathbb{N}$ and $i=0, \ldots, d-1$ write $e_{i}^{n}=\left(n, e_{i}, n\right)$ where $e_{i}$ is the identity of the group $G_{i}$. The elements $e_{i}^{n}$ are the indempotents of $S\left(d, G_{i}, \gamma_{i}\right)$ and we have

$$
e_{0}^{0}>e_{1}^{0}>\cdots>e_{d-1}^{0}>e_{0}^{1}>\cdots>e_{d-1}^{1}>e_{0}^{2}>\cdots .
$$

Hence in the remainder of the paper we will denote a simple regular $\omega$-semigroup by $S\left(d, G_{i}, \gamma_{i}\right)$.

The congruences on $S\left(d, G_{i}, \gamma_{i}\right)$ belonging to the interval $[\iota, \sigma \vee \mathscr{H}]$ were completely described by Baird $[1,2]$ and it was proved that all congruences on $S$ which are not in $[\iota, \sigma \vee \mathscr{H}]$ are group congruences (see [1, Remark p.164]).

We need the following definitions:

DEFINITION 1.2 (see [1, 2 and 3], [2, 2]). Let $S=S\left(d, G_{i}, \gamma_{i}\right)$. A congruence $\mu$ on the set $E(S)$ of indempotents is called uniform if $\left(e_{i}^{n}, e_{j}^{m}\right) \in \mu$ implies that $\left(e_{i}^{n+p}, e_{j}^{m+p}\right) \in \mu$ for all integers $p \geq-\min \{m, n\}$.

Put $G=G_{0} \times G_{1} \times \cdots \times G_{d-1}$, the cartesian product of the $G_{i}$. A subset A of $G$ will be called $\gamma$-admissible if

(i) $A=A_{0} \times \cdots \times A_{d-1}$, for some $A_{i} \subseteq G_{i}, i=0,1, \ldots, d-1$.

(ii) $A_{i} \unlhd G_{i}$, for $i=0,1, \ldots, d-1$.

(iii) $A_{d-1} \gamma_{d-1} \subseteq A_{0}$ and $A_{i} \gamma_{i} \subseteq A_{i+1}$, for $i=0,1, \ldots, d-2$.

If $A=A_{0} \times \cdots \times A_{d-1}$ and $B=B_{0} \times \cdots \times B_{d-1}$ are $\gamma$-admissible subsets of $G$ we define $A \cdot B=A_{0} \cdot B_{0} \times \cdots \times A_{d-1} \cdot B_{d-1}$. Obviously $A \cdot B$ is a $\gamma$-abmissible subset of $G$. Let $\mu$ be a uniform congruence on $E(S), A$ a $\gamma$-admissible subset of $G$ 
and $i$ an integer with $0 \leq i<d$. Put $\mu-\operatorname{rad} A_{i}=\left\{a_{i} \in G_{i} \mid a_{i} \alpha_{n d+i, m d+j} \in A_{j}\right.$ for some positive integers $n, m$ and for some $j$ with $0 \leq j<d$ such that $\left(e_{i}^{n}, e_{j}^{m}\right) \in \mu$ and $\left.e_{j}^{m} \leq e_{i}^{n}\right\}$, and $\mu-\operatorname{rad} A=\mu-\operatorname{rad} A_{0} \times \cdots \times \mu-\operatorname{rad} A_{d-1}$.

If $\mu-\operatorname{rad} A=A, \mu$ and $A$ are called linked.

We remark that the set of the $\gamma$-admissible subsets of $G$ forms a lattice $\Gamma(S)$, with respect to the union gived by the previously defined product and set theoretical intersection (see [1, p. 164]). Moreover, for every uniform congruence $\mu$, the set $\Gamma_{\mu}(S)$ of $\mu$-linked subsets of $G$ is a subsemilattice of $\Gamma(S)$, with respect to intersection. This is a lattice because the $\mu$-linked subsets of $G$ form a partially ordered set isomorphic to the set of congruences in $[\iota, \sigma \vee \mathscr{H}]$ having trace $\mu$ (see [10]).

Let $S=S\left(d, G_{i}, \gamma_{i}\right)$. Subsequently we will use the following notation:

(i) we let 1 be the $\gamma$-admissible subset $\left\{e_{0}\right\} \times \cdots \times\left\{e_{d-1}\right\}$ of $G$;

(ii) for any congruence $\tau$ on $S$, let $A^{\tau}=A_{0}^{\tau} \times \cdots \times A_{d-1}^{\tau}$ where $A_{i}^{\tau}=\left\{a_{i} \in\right.$ $\left.G_{i} \mid\left(0, a_{i}, 0\right) \tau e_{i}^{0}\right\}, i=0, \ldots, d-1$.

The following definition is well known (see [10]).

DEFINITION 1.3. Let $\rho$ be a congruence on an inverse semigroup $S$. The trace of $\rho$, denoted by $\operatorname{tr} \rho$, is the restriction of $\rho$ to the set $E(S)$.

THEOREM C (see [1, Theorems 4.2, 5.1, 5.2, 5.3]). Let $S=S\left(d, G_{i}, \gamma_{i}\right)$, let $\mu$ be a uniform congruence on $E$, let $A$ be a $\gamma$-admissible subset of $G$, and suppose that $\mu$ and $A$ are linked. Then

$$
\begin{aligned}
\tau=\left\{\left(\left(m, a_{i}, n\right),\left(p, b_{j}, q\right)\right) \in S \times S \mid\left(a_{i} \alpha_{u, w}\right)\left(b_{j}^{-1} \alpha_{v, w}\right) \in A_{w(\bmod d)},\right. & \text { where } \\
& \left.u=n d+i, v=q d+j, w=\max \{u, v\} ; m-n=p-q ;\left(e_{i}^{m}, e_{j}^{p}\right) \in \mu\right\}
\end{aligned}
$$

is a congruence on $S$ contained in $[\iota, \sigma \vee \mathscr{H}]$ such that $\operatorname{tr} \tau=\mu$ and $A^{\tau}=A$.

Conversely, let $\tau$ be a congruence on $S=S\left(d, G_{i}, \gamma_{i}\right)$ contained in $[\iota, \sigma \vee \mathscr{H}]$. Then $\tau$ is of the above form with $\mu=\operatorname{tr} \tau$ and $A=A^{\tau}$. Moreover, let $\rho, \lambda$ be congruences on $S=S\left(d, G_{i}, \gamma_{i}\right)$ contained in $[\iota, \sigma \vee \mathscr{H}]$. Then

(i) $\rho \leq \lambda$ if and only if $\operatorname{tr} \rho \leq \operatorname{tr} \lambda$ and $A^{\rho} \subseteq A^{\lambda}$,

(ii) $\operatorname{tr}(\rho \vee \lambda)=\operatorname{tr} \rho \vee \operatorname{tr} \lambda, \operatorname{tr}(\rho \wedge \lambda)=\operatorname{tr} \rho \wedge \operatorname{tr} \lambda$,

(iii) $A^{\rho \vee \lambda}=(\operatorname{tr} \rho \vee \operatorname{tr} \lambda)-\operatorname{rad} A^{\rho} \cdot A^{\lambda}, A^{\rho \wedge \lambda}=A^{\rho} \cap A^{\lambda}$.

We recall the following well-known definitions.

DEFINITION 1.4. Let $L$ be a lattice and let $a, b \in L$. We say that $a$ covers $b$, denoted $a>b$, if $a>b$ and there is no element $c \in L$ such that $a>c>b$.

DEFINITION 1.5 (see [7]). A lattice $L$ is called strongly semimodular if, for every $a, b \in L, a \succ a \cap b$ implies $a \cup b \succ b$. 
We prove the following.

PROPOSITION 1.6. Let $S=S\left(d, G_{i}, \gamma_{i}\right)$. The following are equivalent.

(i) Let $A, B$ be $\gamma$-admissible subsets of $G$. If $B \supseteq A$ and $A$ is $\mu$-linked, then $B$ is $\mu$-linked, for each uniform conqruence $\mu$.

(ii) The interval $[\iota, \sigma \vee \mathcal{H}]$ of $L(S)$ is strongly semimodular.

(iii) The set $\Gamma_{\mu}$ of $\mu$-linked subsets of $G$ is a filter of the lattice $\Gamma$ of $\gamma$-admissible subsets of $G$, for each uniform congruence $\mu$.

(iv) The map $f:[\iota, \sigma \vee \mathcal{H}] \rightarrow \Gamma$ defined by $f(\rho)=A^{\rho}$ is a homomorphism of lattices.

PROOF. (i) implies (ii). Let $\lambda, \rho$, be two congruences in $[\iota, \sigma \vee \mathscr{H}]$ such that $\lambda \succ \lambda \wedge \rho$. Denote by $\nu$ and $\mu$ the traces of $\lambda$ and $\rho$ respectively. We distinguish the following cases:

CASE 1: $v$ and $\mu$ are not comparable, or $\mu<\nu$. Then $\operatorname{tr}(\lambda \wedge \rho) \neq v$ and from [4, Lemma 2.6 (ii)], it follows that $v$ covers $\operatorname{tr}(\lambda \wedge \rho)$ and $A^{\lambda \wedge \rho}=A^{\lambda}$. Hence $A^{\rho} \supseteq A^{\lambda}$. Since $A^{\lambda}$ is $\nu$-linked, by hypothesis $A^{\rho}$ is $\nu$-linked; thus $A^{\rho}$ is $(\nu \vee \rho)$-linked by [3, Lemma 1.3]. For the congruence $\lambda \vee \rho$, we have $A^{\lambda \vee \rho}=(\nu \vee \mu)-\operatorname{rad} A^{\rho} \cdot A^{\lambda}=$ $(\nu \vee \mu)-\operatorname{rad} A^{\rho}=A^{\rho}$ and $\operatorname{tr}(\lambda \vee \rho)=\nu \vee \mu$. It is well known that uniform congruences form a modular lattice; hence $\nu \vee \mu$ covers $\mu$, and so $\lambda \vee \rho$ covers $\rho$ by [4, Lemma 2.5 . (ii)].

CASE 2: $\nu<\mu$. Since $\operatorname{tr}(\lambda \wedge \rho)=\operatorname{tr} \lambda, A^{\lambda}$ covers $A^{\rho} \cap A^{\lambda}$ in the semilattice $\Gamma_{\nu}$ of $\nu$-linked subsets of $G$ (by [4, Lemma 2.5 (1)]). Now let $C$ be a $\gamma$-admissible subset of $G$ containing $A^{\rho} \cap A^{\lambda}$. Then condition (i) implies that $C$ is $\nu$-linked; hence $A^{\lambda}$ covers $A^{\rho} \cap A^{\lambda}$ in the modular lattice $\Gamma$ of $\gamma$-admissible subsets of $G$, and so $A^{\rho} \cdot A^{\lambda}$ covers $A^{\rho}$ in $\Gamma$. Moreover, $A^{\rho}$ is $\mu$-linked, and by (i) $A^{\rho} \cdot A^{\lambda}$ is $\mu$-linked; hence we have $A^{\rho \vee \lambda}=(\nu \vee \mu)-\operatorname{rad} A^{\rho} \cdot A^{\lambda}=A^{\rho} \cdot A^{\lambda}$. Thus, since $\operatorname{tr}(\rho \vee \lambda)=\operatorname{tr} \rho$, it follows that $\lambda \vee \rho$ covers $\rho$, using [4, Lemma 2.5.(i)].

CASE 3: $\mu=v$. The statement immediately follows from the fact that congruences having the same trace form a modular lattice (see [10, Corollary III.2.7]).

(i) implies (iv). Let $\lambda$ and $\rho$ be two congruences in $[\iota, \sigma \vee \mathscr{H}]$ having traces $\nu$ and $\mu$ respectively. From (i) it follows that $A^{\rho} \cdot A^{\lambda}$ is $\nu$-linked and $\mu$-linked, so it is $(\nu \vee \mu)$-linked and $A^{\rho \vee \lambda}=(\nu \vee \mu)-\operatorname{rad} A^{\rho} \cdot A^{\lambda}=A^{\rho} \cdot A^{\lambda}$.

(iv) implies (iii). For all $\mu$-linked subsets $A, B$ of $G$, it is well known that $A \cap B$ is $\mu$-linked. Now, let $\lambda$ be the congruence with trace $\mu$ and $A^{\lambda}=A$, and let $\rho$ be the congruenec with trace $\iota$ and $A^{\rho}=B$. Note that $\lambda$ and $\rho$ are in $[\iota, \sigma \vee \mathscr{H}]$. Hence $A^{\rho \vee \lambda}=(\mu \vee \iota)-\operatorname{rad} A^{\rho} \cdot A^{\lambda}=\mu-\operatorname{rad} A \cdot B$, but (iv) implies that $A^{\rho \vee \lambda}=A \cdot B$ and so $A \cdot B$ is $\mu$-linked.

(iii) implies (i). If $\mathrm{A}$ is $\mu$-linked and $B$ is a $\gamma$-admissible subset containing $A$, then $A \cdot B=B$ is $\mu$-linked by condition (iii). 
(ii) implies (i). Let $A$ be a $\mu$-linked subset and let $B$ be a $\gamma$-admissible subset containing $A$. Now $B$ is $v$-linked for some uniform congruence $v \leq \mu$. Suppose that $v$ is a maximal uniform congruence with $v \leq \mu$, such that $B$ is $\nu$-linked, suppose $\nu<\mu$, and consider a finite chain of uniform congruences $\mu \succ v_{1} \succ v_{2} \succ v_{3} \succ \cdots \succ v_{k}=v$. Then $A$ is $v_{i}$-linked for every $1 \leq i \leq k$. The congruence $\rho$ having trace $v_{k-1}$ and satisfying $A^{\rho}=A$, and the congruence $\lambda$ with $\operatorname{tr} \lambda=\nu$ satisfying $A^{\lambda}=B$, are in $[\iota, \sigma \vee \mathscr{H}]$. The congruence $\rho \wedge \lambda$ has trace $\nu$ and $A^{\rho \wedge \lambda}=A$, and so $\rho \succ \rho \wedge \lambda$. Hence $\lambda \vee \rho \succ \lambda$ and $\operatorname{tr}(\lambda \vee \rho) \neq \operatorname{tr} \lambda$ implies $A^{\rho \vee \lambda}=v_{k-1}-\operatorname{rad} B=A^{\lambda}=B$. Then by [4, Remark 2.4], $B$ is $v_{k-1}$-linked, contradicting the assumption of maximality of $v$. Hence $v=\mu$.

\section{Section 2}

In this section we prove the equivalence between the conditions of modularity and strong semimodularity for the congruence lattice of a simple regular $\omega$-semigroup.

We recall that a lattice $L$ is called modular if for every $a, b, c \in L$ with $a \leq b$, if $a \cup c=b \cup c$ and $a \cap c=b \cap c$, then $a=b$.

LEMMA 2.1. Let $S=S\left(d, G_{i}, \gamma_{i}\right)$. If $[\imath, \sigma \vee \mathscr{H}]$ is strongly semimodular, then $L(S)$ is modular.

PROOF. Since [ $\iota, \sigma \vee \mathscr{H}]$ is strongly semimodular, condition (i) of Proposition 1.6 holds. Let $A$ be a $\gamma$-admissible subset of $G$ and let $\mu$ be a uniform congruence on the idempotents $E(S)$. It is well known that $\mu-\operatorname{rad} 1 \subseteq A \cdot \mu-\operatorname{rad} 1$. Since $A \cdot \mu-\operatorname{rad} 1$ is a $\gamma$-admissible subset and $\mu-\operatorname{rad} 1$ is $\mu$-linked; condition (i) of Proposition 1.6 implies that $A \cdot \mu-\operatorname{rad} 1$ is $\mu$-linked, whence $A \cdot \mu-\operatorname{rad} 1=\mu-\operatorname{rad}(A \cdot \mu-\operatorname{rad} 1) \supseteq \mu-\operatorname{rad} A$. Thus $A \cdot \mu-\operatorname{rad} 1=\mu-\operatorname{rad} A$ and $L(S)$ is modular by [3, Theorem 1.8].

THEOREM 2.2. Let $S=S\left(d, G_{i}, \gamma_{i}\right)$.The congruence lattice $L(S)$ of $S$ is modular if and only if it is strongly semimodular.

PROOF. It is well known that $L(S)$ being modular implies that $L(S)$ is strongly semimodular.

Suppose that $L(S)$ is strongly semimodular. Then $[\iota, \sigma \vee \mathscr{H}]$ is strongly semimodular because it is a convex sublattice of $L(S)$ (see for instance [11, Lemma 2.2]), and the statement follows from Lemma 2.1.

We recall the following 
DEFINITION 2.3. A lattice $L$ is called $M$-symmetric if the modularity relation $M$, defined by $a M b$ if and only if for every $x \in[a \cap b, b]$ we have $x=(x \cup a) \cap b$, is symmetric.

From the above result we obtain the following

COROLlary 2.4. Let $S=S\left(d, G_{i}, \gamma_{i}\right)$. The following are equivalent.

(i) $L(S)$ is modular.

(ii) $L(S)$ is $M$-symmetric.

(iii) $L(S)$ is strongly semimodular.

PROOF. This result immediately follows from the well known fact that every modular lattice is $M$-symmetric and every $M$-symmetric lattice is strongly semimodular.

REMARK 2.5. Among the conditions similar to modularity quoted in $[6, p .6]$ the only one strictly weaker than modularity for the congruence lattice of a regular $\omega$ semigroup is semimodularity.

We recall that a lattice $L$ is called semimodular if, for every $a, b \in L$ such that $a$ and $b$ both cover $a \cap b$, then $a \cup b$ covers $a$ and $b$. The authors studied this condition in [4].

\section{Section 3}

When we establish modularity conditions for the congruence lattice of a simple regular $\omega$-semigroup $S$, we need consider only suitable sublattices of the congruence lattice of $S$.

First we prove the following.

LEMMA 3.1. Let $S=S\left(d, G_{i}, \gamma\right)$. The following are equivalent.

(i) Let $A, B$ be $\gamma$-admissible subsets of $G$ and let $\mu$ be a uniform congruence $\mu$ covering $\iota$. If $B \supseteq A$ and $A$ is $\mu$-linked, then $B$ is $\mu$-linked.

(ii) Let $A, B$ be $\gamma$-admissible subsets of $G$ and let $\mu$ be a uniform congruence. If $B \supseteq A$ and $A$ is $\mu$-linked, then $B$ is $\mu$-linked.

PROOF. It is immediate that (ii) implies (i).

(i) implies (ii). Suppose $v$ is a uniform congruence which does not cover $\iota$; then $\nu=v_{1} \vee v_{2} \vee \cdots \vee v_{h}$ with $v$ uniform congruences covering $\iota$. Now let $A, B$ be two $\gamma$-admissible subsets of $G$, and suppose that $B \supseteq A$ and that $A$ is $v$-linked. Then $A$ is $v_{i}$-linked for every $1 \leq i \leq h$, and so $B$ is $v_{i}$-linked from the hypothesis and by [3, Lemma 1.3] it follows that $B$ is $\left(v_{1} \vee v_{2} \vee \cdots \vee v_{h}\right)$-linked. 
THEOREM 3.2. Let $\mu$ be any uniform congruence of simple regular $\omega$-semigroup $S$ covering $\iota$ in the lattice of uniform congruences. Let $\sigma_{\mu}$ be the least congruence having trace $\mu$. Then $L(S)$ is modular if and only if $\left[\mathscr{H} \wedge \sigma_{\mu}, \mathscr{H} \vee \sigma_{\mu}\right]$ is strongly semimodular.

PROOF. [ $\mathscr{H} \wedge \sigma_{\mu}, \mathscr{H} \vee \sigma_{\mu}$ ] is a sublattice of $L(S)$, so if $L(S)$ is modular, it is also modular and obviously strongly semimodular. Now let $\left[\mathscr{H} \wedge \sigma_{\mu}, \mathscr{H} \vee \sigma_{\mu}\right]$ be a strongly semimodular lattice. In view of Lemma 2.1 it is enough to prove that $[\iota, \sigma \vee \mathscr{H}]$ is strongly semimodular. Suppose that $\mu$ is a uniform congruence covering $\iota$, that $A$ and $B$ are $\gamma$-admissible subsets with $B \supseteq A$, and that $A$ is $\mu$-linked. Let $\lambda$ be the congruence having trace $\iota$ and $A^{\lambda}=B$, and let $\rho$ be the congruence having trace $\mu$ and $A^{\mu}=B$. Both the congruences are in $\left[\mathscr{H} \wedge \sigma_{\mu}, \mathscr{H} \vee \sigma_{\mu}\right]$. In fact $\mathscr{H} \wedge \sigma_{\mu}$ has trace $\iota, A^{\mathscr{H} \wedge \sigma_{\mu}}=\operatorname{rad} 1, \mathscr{H} \vee \sigma_{\mu}$ has trace $\mu$ and $A^{\mathscr{H} \vee \sigma_{\mu}}=G$. Thus $\lambda \wedge \rho$ has trace $\iota, A^{\lambda \wedge \rho}=A$ and $\rho$ covers $\lambda \wedge \rho$. From the hypothesis it follows that $\lambda \vee \rho$ cover $\lambda$. But $\lambda \vee \rho$ has trace $\mu$ and $A^{\lambda \vee \rho}=\mu-\operatorname{rad} B$, so $\mu-\operatorname{rad} B=B$ [4, Remark 2.4], and the statement follows from the previous lemma and Proposition 1.6.

\section{Section 4}

The following definition is well-known (see [10]).

DEFINITION 4.1. Let $S$ be an inverse semigroup. A full inverse subsemigroup $K$ of $S$ is called a kernel in $S$ if it satisfies $a b \in K$ implies $a K b \subseteq K(a, b \in S)$. Denote by $K(S)$ the set of kernels in $S$ ordered by inclusion. The kernel map is defined by $\kappa: \rho \rightarrow \operatorname{ker} \rho$. The map $\kappa$ is a complete $\cap$-homomorphism of $L(S)$ onto $K(S)$. The equivalence relation $\mathscr{K}$ on $L(S)$ induced by $\kappa$ is called the kernel relation.

The relation $\mathscr{K}$ is a congruence with respect to intersection but in general it is not a congruence on $L(S)$ (see either [10, Ex.III.4.11], or [8], or [9])

We prove the following

THEOREM 4.2. Let $S=S\left(d, G_{i}, \gamma_{i}\right) . L(S)$ is modular if and only if $\mathscr{K}$ is a congruence on $L(S)$.

PROOF. Suppose that $L(S)$ is modular and let $\lambda, \rho$ be two congruence on $S$ such that $\lambda \mathscr{K} \rho$. Let $\tau$ be a congruence on $S$. We have to prove that $\lambda \vee \tau \mathscr{K} \rho \vee \tau$. Firstly we prove that $\operatorname{ker}(\rho \vee \tau)=\operatorname{ker} \rho \vee \operatorname{ker} \tau$ for every congruence $\rho, \tau$ on $S$. It is immediate that $\operatorname{ker}(\rho \vee \tau) \supseteq \operatorname{ker} \rho \vee \operatorname{ker} \tau$. In order to prove that $\operatorname{ker}(\rho \vee \tau) \subseteq \operatorname{ker} \rho \vee \operatorname{ker} \tau$ we distinguish two cases:

CASE 1. Both the congruences $\rho, \tau$ are in $[\iota, \sigma \vee \mathscr{H}]$. Firstly remark that from the definition of $A^{\rho}$, it follows immediately that $\operatorname{ker} \rho=\left\{(m, a, m) \mid m \in \mathbb{N}, a \in A^{\rho}\right\}$ 
for every congruence $\rho \in[\iota, \sigma \vee \mathscr{H}]$. Hence $\rho \vee \tau \in[\iota, \sigma \vee \mathscr{H}]$ gives $\operatorname{ker}(\rho \vee \tau)=$ $\left\{(n, c, n) \mid n \in \mathbb{N}, c \in A^{\rho \vee \tau}\right\}$. Moreover Proposition 1.6 implies that that $A^{\rho \vee \tau}=$ $A^{\rho} \cdot A^{\tau}$. Since $\operatorname{ker} \rho \vee \operatorname{ker} \tau$ contains the set $\left\{(m, a b, m) \mid m \in \mathbb{N}, a \in A^{\rho}, b \in A^{\tau}\right\}$ we immediately deduce that $\operatorname{ker}(\rho \vee \tau) \subseteq \operatorname{ker} \rho \vee \operatorname{ker} \tau$.

CASE 2. At least one of the congruences $\rho, \tau$ is not in $[\iota, \sigma \vee \mathscr{H}]$. Let $\tau \notin$ $[\iota, \sigma \vee \mathscr{H}]$. Then $\tau$ is a group congruence. Since $\operatorname{ker}(\sigma \vee \mathscr{H}) \nsupseteq \operatorname{ker} \rho \vee \operatorname{ker} \tau$, $\operatorname{ker} \rho \vee \operatorname{ker} \tau$ is a kernel of a congruence $\theta \notin[\iota, \sigma \vee \mathscr{H}]$. Hence $\theta$ is a group congruence greater than $\rho$ and $\tau$, so $\theta \geq \rho \vee \tau$ and $\operatorname{ker} \theta \supseteq \operatorname{ker}(\rho \vee \tau)$.

Now it immediately follows that $\mathscr{K}$ is also a congruence with respect to the join.

Conversely let $\mathscr{K}$ be a congruence. Let $B$ and $A$ be $\gamma$-admissible subgroups such that $B \supseteq A$ and let $A$ be $\mu$-linked for some uniform congruence $\mu$. Let $\rho, \lambda, \tau$ be three congruences in $[\iota, \sigma \vee \mathscr{H}]$ such that $\operatorname{tr} \lambda=\iota, \operatorname{tr} \rho=\mu, \operatorname{tr} \tau=\iota$ and $A^{\lambda}=A$, $A^{\rho}=A, A^{\tau}=B$. Thus we have $\lambda \mathscr{K} \rho$. Hence $\lambda \vee \tau \mathscr{K} \rho \vee \tau$. Since both $\lambda \vee \tau$ and $\rho \vee \tau$ are in $[\iota, \sigma \vee \mathscr{H}]$, we have $\operatorname{ker}(\lambda \vee \tau)=\left\{(m, a, m) \mid m \in \mathbb{N}, a \in A^{\lambda \vee \tau}\right\}$ and $\operatorname{ker}(\lambda \vee \tau)=\left\{(m, a, m) \mid m \in \mathbb{N}, a \in A^{\rho \vee \tau}\right\}$, but $A^{\lambda \vee \tau}=A \cdot B=B$ and $A^{\rho \vee t}=\mu-\operatorname{rad} A \cdot B=\mu-\operatorname{rad} B$. Thus $\mu-\operatorname{rad} B=B$ and $L(S)$ is modular from Proposition 1.6 and Lemma 2.1.

We extend the previous results in the following.

THEOREM 4.3. Let $S=S\left(d, G_{i}, \gamma_{i}\right)$. The following conditions are equivalent.

(1) $L(S)$ is modular.

(2) $L(S)$ is strongly semimodular.

(3) $L(S)$ is $M$-symmetric.

(4) $\mathscr{K}$ is a congruence on $L(S)$.

Moreover all these equivalent properties are equivalent to the same properties on sublattices of $L(S)$. In fact the following theorem holds.

THEOREM 4.4. Let $S=S\left(d, G_{i}, \gamma_{i}\right)$. Let $\mu$ be any uniform congruence covering । and let $\sigma_{\mu}$ be the least congruence having trace $\mu$. The following are equivalent.

(1) $\left[\mathscr{H} \wedge \sigma_{\mu}, \mathscr{H} \vee \sigma_{\mu}\right]$ is modular.

(2) $\left[\mathscr{H} \wedge \sigma_{\mu}, \mathscr{H} \vee \sigma_{\mu}\right]$ is strongly semimodular.

(3) $\left[\mathscr{H} \wedge \sigma_{\mu}, \mathscr{H} \vee \sigma_{\mu}\right]$ is $M$-svmmetric

(4) $\mathscr{K}$ is a congruence on $\left[\mathscr{H} \wedge \sigma_{\mu}, \mathscr{H} \vee \sigma_{\mu}\right]$.

(5) $L(S)$ is modular.

In Theorem 3.2 we proved the equivalence between (5) and (2). The other statements can be proved in an analogous way. 
DEFINITION 4.5. For each congruence $\rho$ on $S, \rho^{K}$ denotes the greatest congruence having $\operatorname{ker} \rho$ as kernel.

For each uniform congruence $\mu, \sigma_{\mu}$ denotes the least congruence having trace $\mu$.

We can also prove the following

PROPOSITION 4.6. Let $S=S\left(d, G_{i}, \gamma_{i}\right)$. Let $\mu$ be a uniform congruence covering $\iota$. Then $L(S)$ is modular if and only if for every congruence $\rho$ with $\mathscr{H} \wedge \sigma_{\mu} \leq \rho \leq \mathscr{H}$, $\rho^{K}$ contains $\sigma_{\mu}$.

PROOF. Let $\mu$ be a uniform congruence covering $\iota$. Suppose that $\mathscr{H} \wedge \sigma_{\mu} \leq \rho \leq \mathscr{H}$ implies $\rho^{K} \geq \sigma_{\mu}$. Let $B$ and $A$ be two $\gamma$-admissible subgroups with $B \supseteq A$ and let $A$ be $\mu$-linked. The congruence $\rho$ has trace $\iota$ and $A^{\rho}=B$ contains $\mathscr{H} \wedge \sigma_{\mu}$. The congruence $\rho^{K}$ is in $[\imath, \sigma \vee \mathscr{H}]$ because $\operatorname{tr} \rho^{K}=\omega_{E}=\operatorname{tr}(\sigma \vee \mathscr{H})$ and $\operatorname{ker} \rho^{K}=$ $\{(m, B, m) \mid m \in \mathbb{N}\} \subseteq\{(m, G, m) \mid m \in \mathbb{N}\}=\operatorname{ker}(\sigma \vee \mathscr{H})$. So $\rho^{K}$ has trace $\nu$ and $A^{\rho^{K}}=B$ is $\nu$-linked. Since $\rho^{K} \geq \sigma_{\mu}, v \geq \mu$ and $B$ is $\mu$-linked by [4, Remark 2.4]. Hence $L(S)$ is modular. Conversely let $L(S)$ be modular and let $\rho$ belong to [ $\mathscr{H} \wedge \sigma_{\mu}, \mathscr{H}$ ] for a uniform congruence $\mu$ covering $\iota$. Then $\rho$ has trace $\iota$ and $A^{\rho}=B$ with $B \supseteq \mu-\operatorname{rad} 1$. Now $\mu-\operatorname{rad} 1$ is $\mu$-linked and by hypothesis $\mathrm{B}$ is also $\mu$-linked. Let $v$ be the trace of $\rho^{K}, v \geq \mu$; since $\operatorname{ker} \rho^{K}=\{(m, B, m) \mid m \in \mathbb{N}\}$, we deduce $\rho^{K} \geq \sigma_{\mu}$.

\section{Section 5}

Now we examine the general case of regular $\omega$-semigroups.

It is well-known that a non-simple regular $\omega$-semigroup $S$ is either an $\omega$-chain of groups or the disjoint union of a finite chain of groups $H=\left[n, H_{j}, \phi_{j}\right]$ and a simple regular $\omega$-semigroup $K=K\left(d, K_{i}, \psi_{i}\right)$ which is an ideal of $S$. In the latter case, as in $[3,4]$, we will use the notation $S=S\left(\left[n, H_{j}, \phi_{j}\right] ; K, \phi\right)$ where $\phi$ is the homomorphism which induces the retract extension of $K$ by $H^{0}$, and which is actually a homomorphism of $H$ into $K_{0}$.

Modularity, $M$-symmetry and strong semimodularity of the congruence lattice of $\omega$-chains $\left[\Omega, H_{j}, \phi_{j}\right]$ of groups are equivalent to the triviality of $\phi_{j}$ for every $j \in \mathbb{N}$ (see, for instance [11, Th.6.5 and Th.6.6]).

For a regular $\omega$-semigroup $S=S\left(\left[n, H_{j}, \phi_{j}\right] ; K, \phi\right)$ we deduce from [11, Theorem 6.6] that $L(S)$ is modular if and only if $K$ has a modular lattice of congruences and $\phi$ and all $\phi_{j}$ are trivial. Moreover if $L(S)$ is strongly semimodular then $\phi$ and all $\phi_{j}$ are trivial from [11, Theorem 6.5] and $K$ is strongly semimodular. Then we immediately deduce from Corollary 2.4 that $M$-symmetry and strong semimodularity of the congruence lattice of $S=S\left(\left[n, H_{j}, \phi_{j}\right] ; K, \phi\right)$ are equivalent to modularity. 
As concerns the relation $\mathscr{K}$, Petrich [9] proved the following

THEOREM D. Let $\left[Y ; S_{\alpha}, \phi_{\alpha, \beta}\right]$ be a strong semilattice of simple regular semigroups. $\mathscr{K}$ is a congruence if and only if

(1) $\mathscr{K}$ is a congruence on $L\left(S_{\alpha}\right)$ for every $\alpha \in Y$,

(2) $S_{\alpha} \phi_{\alpha, \beta} \subseteq E\left(S_{\beta}\right)$ whenever $\alpha>\beta$.

Then we easily deduce the following

THEOREM 5.1. Let $S$ be a regular $\omega$-semigroup. The following conditions are equivalent

(1) $L(S)$ is modular.

(2) $L(S)$ is strongly semimodular.

(3) $L(S)$ is $M$-symmetric.

(4) $\mathscr{K}$ is a congruence on $L(S)$.

PROOF. We just observed the equivalence of conditions (1), (2), (3). If (1) holds then (4) follows from Theorem D and Theorem 4.3.

Suppose that (4) holds. Then if $S$ is simple, (1) follows from Theorem 4.3. If $S$ is an $\omega$-chain of groups Theorem D implies that $\phi_{j}$ are trivial for every $j \in \mathbb{N}$, whence (1) follows. If $S=S\left(\left[n, H_{j}, \phi_{j}\right] ; K, \phi\right)$, from Theorem D we deduce that $H_{n-1} \phi \subseteq E\left(K_{0}\right)$ and $H_{j} \phi_{j} \subseteq E\left(H_{j+1}\right)$ for $0 \leq j \leq n-2$, whence $\phi$ and $\phi_{j}$ are trivial. Moreover Theorem $\mathrm{D}$ implies that $\mathscr{K}$ is a congruence on $L(K)$. So by Theorem 4.3, $L(K)$ is modular and $L(S)$ is modular by [3, Lemma 3.4].

\section{Section 6}

Now we compare the condition of modularity for $L(S)$, with the condition of $\theta$-modularity of $S$. We recall the following

DEFINITION 6.1 ([12]). A regular semigroup $S$ is called $\theta$-modular if for all congruences $\lambda, \rho, \tau$ on $S$, conditions $\lambda \wedge \rho=\lambda \wedge \tau, \lambda \vee \rho=\lambda \vee \tau, \rho \geq \tau$ and $\operatorname{tr} \rho=\operatorname{tr} \tau$ imply $\rho=\tau$.

We can prove the following

LEMMA 6.2. Let $S$ be a regular semigroup, with a modular lattice of congruence traces. If $S$ is $\theta$-modular then $L(S)$ is modular.

PrOOF. Let $S$ be $\theta$-modular, and suppose that there exist three congruences $\lambda, \rho, \tau$ on $S$ with $\lambda \wedge \rho=\lambda \wedge \tau, \lambda \vee \rho=\lambda \vee \tau, \rho \geq \tau$ and $\operatorname{tr} \rho>\operatorname{tr} \tau$. Since in a regular 
semigroup every congruence is uniquely determined by the pair of its trace and kernel, it follows that $\operatorname{tr} \lambda$ is neither comparable with $\operatorname{tr} \rho$ nor with $\operatorname{tr} \tau$. So we have three congruences with $\operatorname{tr} \rho>\operatorname{tr} \tau, \operatorname{tr}(\lambda \wedge \rho)=\operatorname{tr}(\lambda \wedge \tau)$, and $\operatorname{tr}(\lambda \vee \rho)=\operatorname{tr}(\lambda \vee \tau)$, which contradicts the modularity of the lattice of congruence traces.

PROPOSITION 6.3. For a regular $\omega$-semigroup $S$ the following are equivalent.

(i) $L(S)$ is modular.

(ii) $S$ is $\theta$-modular.

PROOF. It is obvious that $L(S)$ modular implies $S$ is $\theta$-modular. Now, let $S$ be $\theta$-modular. The traces, being a sublattice of the congruences lattice on a chain, form a modular lattice and the statement follows from the previous lemma.

REMARK 6.4. Notice that a result analogous to Theorem 4.4 can be proved for $\theta$ modularity. Actually $\theta$-modularity of a simple regular $\omega$-semigroup $S$ can be proved to be equivalent to the following condition: let $\mu$ be a uniform congruence covering $\iota$. Then for all congruences $\lambda, \rho, \tau \in\left[\mathscr{H} \wedge \sigma_{\mu}, \mathscr{H} \vee \sigma_{\mu}\right]$, relations $\lambda \wedge \rho=\lambda \wedge \tau$, $\lambda \vee \rho=\lambda \vee \tau, \rho \geq \tau$ and $\operatorname{tr} \rho=\operatorname{tr} \tau$ imply $\rho=\tau$.

\section{References}

[1] G. R. Baird, 'Congruences on simple regular $\omega$-semigroups', J. Austral. Math. Soc. (Ser. A) 14 (1972), 155-167.

[2] — , 'On a sublattice of the lattice of congruences on a simple regular $\omega$-semigroup', J. Austral. Math. Soc. (Ser. A) 13 (1972), 461-471.

[3] C. Bonzini and A. Cherubini, 'Modularity of the lattice of congruences of a regular $\omega$-semigroup', Proc. Edinburgh Math. Soc. (2) 33 (1990), 405-417.

[4] _ 'Semimodularity of the congruence lattice on regular $\omega$-semigroups', Mh. Mat. 109 (1990), 205-219.

[5] B. P. Kocin, 'The structure of inverse ideally simple $\omega$-semigroups', Vestnik Leningrad Univ. Math. 23 (1968), 41-50.

[6] H. Mitsch, 'Semigroups and their lattice of congruences', Semigroup Forum 26 (1983), 1-63.

[7] W. D. Munn, 'Regular $\omega$-semigroups', Glasgow Math. J. 9 (1968), 46-66.

[8] M. Petrich, 'The kernel relation for a retract extension of Brandt semigroups', preprint.

[9] — 'The kernel relation for certain regular semigroups', preprint.

[10] - Inverse semigroups (Wiley, New York, 1984).

[11] 37 (1988), 167-199.

[12] C. Spitznagel, 'The lattice of congruences on a band of groups', Glasgow Math. J. 14 (1973), 189-197.

Universitá

Politecnicó

Via Saldini 50

Piazza L. ad Vinci 32

20133 Milano

20133 Milano

Italia

Italia

email: bonzini@vmimat.mat.unimi.it

email: aleche@ipmma1.polimi.it 